\title{
Zonas prioritarias de investigación en base a análisis de sesgos de muestreo en la colección de mamíferos de QCAZ
}

\author{
Santiago F. Burneo y Ma. Alejandra Camacho
}

Museo de Zoología. Pontificia Universidad Católica del Ecuador

RESUMEN: La colección de mamíferos del QCAZ presenta sesgos relacionados con el esfuerzo de muestreo que indican una tendencia a realizar colecciones en zonas de fácil acceso. Se encontraron diferencias altamente significativas entre la cantidad de registros colectados en el Sistema Nacional de Áreas Protegidas y fuera de éste. Además, 20 de las 35 áreas del SNAP carecen de colecciones. Tomando en cuenta estos sesgos, puede ser difícil, e incluso erróneo, obtener estimaciones de biodiversidad reales y asignar áreas prioritarias de conservación. La proyección e interpolación de los puntos de recolección en mapas generados por SIG permitió mostrar vacíos en las colecciones, de manera que además de las áreas del SNAP, se proponen zonas prioritarias de colección.

PALABRAS CLAVE: áreas prioritarias, colecciones, mamíferos, museos de historia natural, sesgos, Sistemas de Información Geográfica.

\begin{abstract}
QCAZ mammal's collection presents biases related to sampling effort that show a trend towards collecting in easily access areas. Highly significant differences between amounts of collected records within the National System of Protected Areas and outside of it were found. Besides, 20 out of the 35 protected areas lack collections. Because of these biases, it may be difficult, or even erroneous, to obtain accurate biodiversity assessments or assign conservation priority areas. The projection and interpolation of collection locality points on maps generated by GIS allowed to display gaps in collections efforts, resulting in priority collection areas, that are proposed in addition to the national protected areas.
\end{abstract}

Key words: Biases, collections, Geographic Information Systems, mammals, natural history museums, priority areas. 


\section{INTRODUCCIÓN}

La colección de mamíferos del Museo de Zoología de la Pontificia Universidad Católica del Ecuador (QCAZ) almacena aproximadamente 9000 especímenes, conservados como individuos enteros en solución de alcohol, pieles, cráneos y esqueletos secos, tejidos y transparentaciones. La información de los ejemplares se almacena en una base de datos electrónica que incluye información taxonómica, geográfica, de colección, de desarrollo, morfométrica, ecológica y de existencia de fotografías o tejidos.

Los ejemplares de una colección científica constituyen registros de una especie particular localizada en un espacio y tiempo determinados, que almacenan dos tipos de información: la referencia taxonómica y los datos espaciales. (1) Si es manejada apropiadamente, la información espacial de los registros de una colección científica permite realizar diversos análisis utilizando herramientas de Sistemas de Información Geográfica (SIG).

Sin embargo, en muchos museos de historia natural, la información espacial de los registros de colección es mal manejada y su almacenamiento en bases de datos electrónicas es incompleto y poco accesible, lo que constituye un obstáculo que limita evaluaciones e investigaciones sobre biodiversidad. (2) El manejo, a veces inadecuado, de la información espacial no es el único problema de este tipo que afrontan las colecciones científicas. En ocasiones, importante información espacial no es accesible debido a que las colecciones presentan esfuerzos de muestreo de irregular distribución geográfica.
Los SIG son un conjunto de herramientas que permiten el almacenamiento, manejo y análisis de información geográfica y datos asociados (3), en este estudio, varios análisis SIG han permitido la evaluación geográfica del esfuerzo de muestreo de la colección de mamíferos del QCAZ. Idealmente, el esfuerzo de muestreo debería ser uniforme en un territorio, para evitar variaciones irreales en patrones de distribución de cierta especie. (4) Las colecciones pueden estar sesgadas espacialmente ya que mucho del esfuerzo de colección está localizado en la proximidad de caminos, ríos, estaciones científicas, instituciones o ciudades. $(5,6)$

Otro tipo de sesgo es la colección de taxa de interés para los investigadores (4), quienes gracias a datos obtenidos de la historia natural de algún taxón, se dirigen a los lugares donde esperan encontrar los grupos de su interés, sin exploración de lugares diferentes, o simplemente prefieren lugares convenientemente accesibles. Adicionalmente, las colecciones pueden estar sesgadas por lo que Soberón y colaboradores (1) llaman "moda taxonómica" donde los esfuerzos están centrados en taxa bien conocidos y fáciles de capturar. Teniendo en cuenta esta situación, cualquier análisis utilizando datos espaciales debe ser precedido por la estimación de la cantidad y el tipo de sesgos que presenta una colección. (7)

Existen métodos diseñados para reducir sesgos en la información generada por los registros de una colección científica. Las técnicas de modelamiento son métodos útiles que permiten predecir la distribución de una especie al integrar datos de ocurrencia (registros de colección) con variables 
ambientales y ecológicas, evaluando la idoneidad del hábitat aun para sitios en donde no hay registros de colección $(8,9,10)$. Sin embargo, los sesgos encontrados en la información de una colección nunca estarán completamente superados a menos que se realicen mayores esfuerzos de muestreo en áreas poco estudiadas. (7)

En el Ecuador, hasta la fecha, 35 áreas conforman el Sistema Nacional de Áreas Protegidas (SNAP), que han sido establecidas con el fin de preservar el ambiente, recursos genéticos y vida silvestre del país (Art. 168 del Régimen Forestal, TULAS, 2004). De estas 35 áreas, 33 se encuentran dentro del territorio nacional continental, cubriendo aproximadamente el $16,2 \%$ de su extensión. (11) Sin embargo, el conocimiento de la biodiversidad de estas zonas parece estar poco apoyado por registros de colección, siendo zonas con esfuerzos de colección muy pobres. (12)

El presente trabajo pretende analizar, revisar, completar y, si es necesario, corregir la información asociada a los especímenes para hacer de la colección una fuente confiable de investigación y consulta. Utilizando un conjunto de datos correctos se pueden generar mapas de los sesgos espaciales de la colección. La proyección de los puntos de colección en mapas permite mostrar vacíos en la colección de mamíferos del QCAZ, de manera que se pueden planificar eficazmente futuros esfuerzos de muestreo e incrementar el conocimiento de la mastofauna del país.

\section{MATERIALES Y MÉTODOS}

Se utilizaron 7834 registros de localidades de colección correspondientes a los ejemplares ingresados en la base de datos hasta agosto de 2006. Se excluyeron 201 registros con localidades desconocidas, poco informativas o erróneas y se obtuvo una muestra de 7633 registros con localidades cuya información geográfica es correcta. Los datos que carecían de coordenadas geográficas fueron georreferenciados basándose en la información del lugar. Se obtuvieron 384 localidades únicas georreferenciadas que fueron proyectados en un mapa para revisar la correcta asignación de coordenadas (Figura 1).

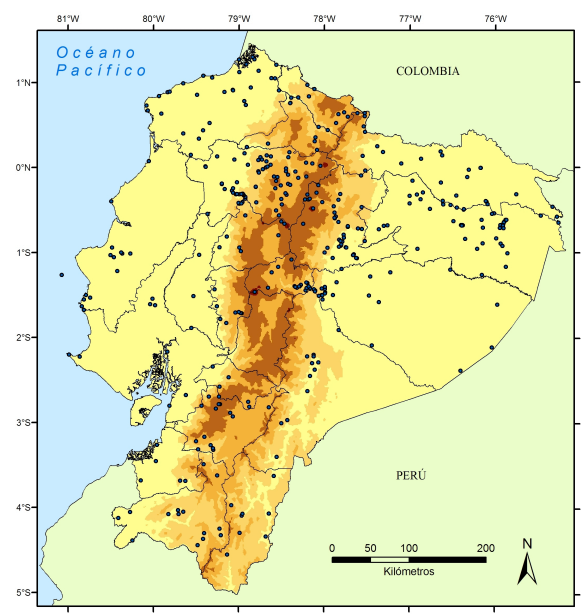

Figura 1. Mapa de localidades únicas de la colección de mamíferos del QCAZ.

Se escogieron las capas con rasgos geográficos de mayor accesibilidad. Los mapas de centros poblados, ríos y vías que se utilizaron para los análisis de las localidades provienen del Proyecto BINU. (13) Además, se usó la capa de ciudades del Almanaque Electrónico Ecuatoriano. (14)

En el caso de los ríos se escogieron aquellos de cuarto, quinto, sexto y séptimo orden, por su navegabilidad (Figura 2a); en el caso de vías, aquellas de primero, segun- 
A
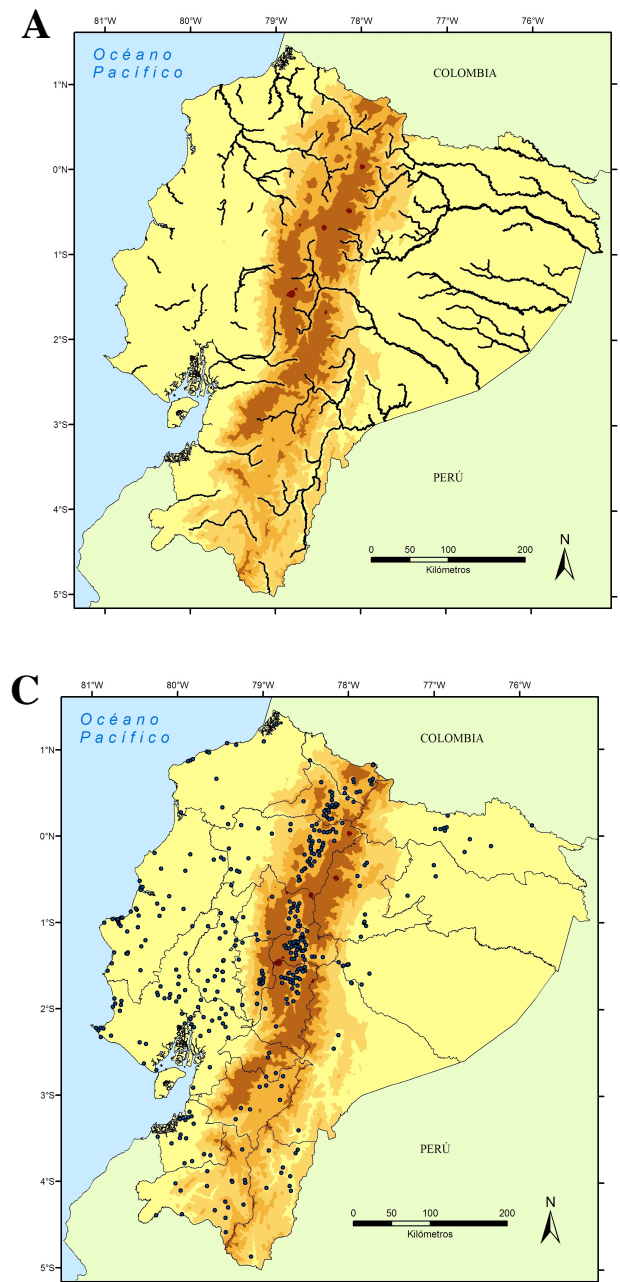

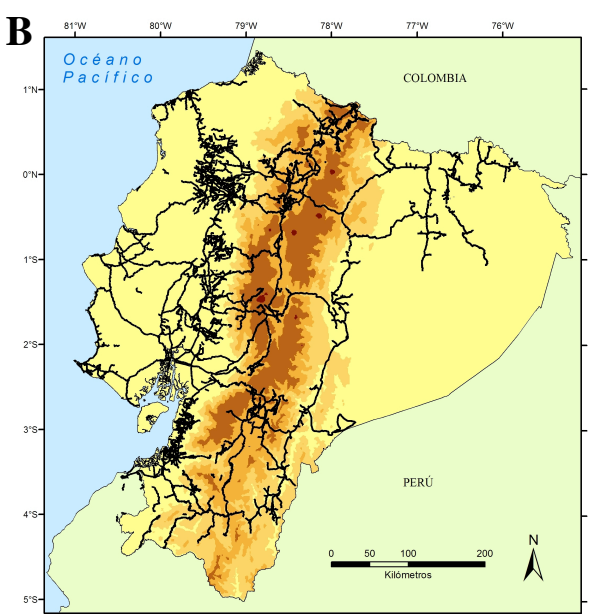

D

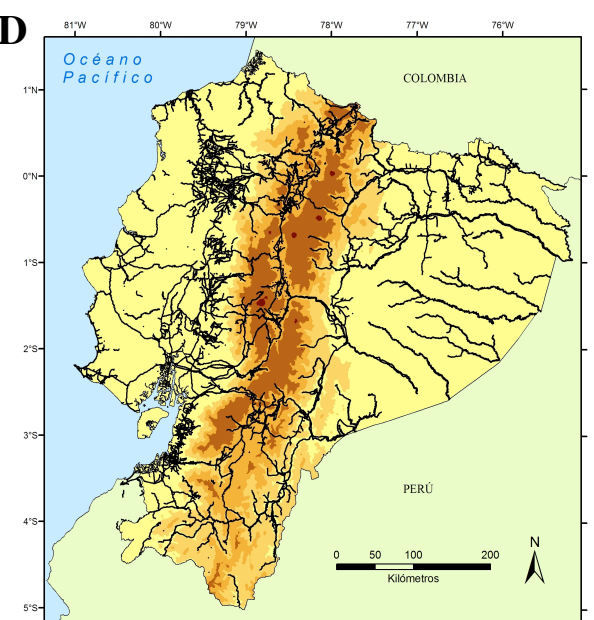

Figura 2. a) Ríos navegables del Ecuador. b) Vías importantes del Ecuador. c) Centros poblados mayores a 100 $\mathrm{km}^{2}$ del Ecuador. d) Rasgos geográficos de mayor accesibilidad.

do y tercer orden, por ser las más importantes (Figura 2b); y, en el caso de poblados se escogieron aquellos con superficies mayores a $100 \mathrm{~km}^{2}$, por presentar mayores facilidades para investigación (Figura 2c).

Siguiendo la metodología de Reddy y Dávalos (6), se generaron el mismo número de puntos aleatorios que las localidades únicas obtenidas. Se exploraron sesgos debidos a la accesibilidad midiendo la proximidad de cada registro de colección a centros poblados, ríos y vías independientemente (6) y, además, de cada registro de colección y punto aleatorio a cualquiera de los rasgos antes mencionados, al crear una capa que incluya como polígonos tanto a los centros poblados, como a ríos y vías (Figura 2d). 
Los análisis de proximidad se realizaron utilizando la función "Analysis Tools: Near" de ArcToolbox - ArcGIS 8.1 (15) y se consideró un radio de $200 \mathrm{~km}$ alrededor de cada punto. Se utilizó la prueba estadística de Kolmogorov-Smirnov para determinar si existe una diferencia significativa en la distribución de las muestras analizadas. $(16,17)$

Se realizó un análisis para determinar si existe un sesgo en la realización de colecciones de mamíferos en el Sistema Nacional de Áreas Protegidas, mediante dos pruebas estadísticas de Ji cuadrado $\left({ }^{2}\right.$ ) para evaluar si el esfuerzo de captura ha sido regularmente distribuido en todo el territorio nacional. Se hicieron dos pruebas de Ji cuadrado $\left({ }^{2}\right)$ con dos metodologías independientes para evaluar este posible sesgo $(18,19)$.

En la primera prueba los datos observados corresponden a los registros de colección dentro y fuera del SNAP. Los primeros fueron determinados utilizando la función
"Select by location: Are completely within" de ArcMap 8.1; (15) los registros de colección fuera del SNAP corresponden a los datos restantes. Los datos esperados son los posibles registros de colección dentro y fuera del SNAP suponiendo que el esfuerzo de colección fuera proporcional al territorio que ocupan las zonas que conforman el SNAP y las zonas no protegidas del país. Para una segunda prueba ${ }^{2}$ se consideró que si los ejemplares han sido colectados sin sesgos en el territorio nacional, la cantidad de registros colectados dentro y fuera del SNAP (datos observados) sería proporcional a la cantidad de puntos aleatorios generados por herramientas de SIG, igualmente, dentro y fuera de las áreas protegidas (datos esperados).

Para el análisis de interpolación en el programa ArcGis 8.1 se corrió la opción "Inverse Distance Weighted" (IDW) de la función "Spatial Analyst: Interpolate to raster". Mediante este método de interpolación, se obtiene una capa tipo cuadrícula,

Tabla 1. Cantidad y proporción de localidades de colección y puntos aleatorios en los diferentes rangos de distancias a los poblados (A), ríos (B) o vías (C) más cercanos. Las clases representan rangos de distancias transformadas a su logaritmo natural $(>0-\leq 1=>0 \mathrm{~m}-\leq 10 \mathrm{~m} ;>1-\leq 2=>10$ $\mathrm{mm}-\leq 100 \mathrm{~m} ;>2-\leq 3=>100 \mathrm{~m}-\leq 1 \mathrm{~km} ;>3-\leq 4=>1 \mathrm{~km}-\leq 10 \mathrm{~km} ;>4-\leq 5=>10 \mathrm{~km}-\leq 100$ $\mathrm{km} ;>5-\leq 6=>100 \mathrm{~km})$.

\begin{tabular}{|ccccc|}
\hline A. Poblados & \multicolumn{2}{c}{ Puntos localidades verdaderas } & \multicolumn{2}{c|}{ Puntos aleatorios } \\
\hline $\begin{array}{c}\text { Clases } \\
\text { Log } 10)\end{array}$ & $\begin{array}{c}\text { Frecuencias } \\
\text { absolutas }\end{array}$ & $\begin{array}{c}\text { Frecuencias } \\
\text { relativas }\end{array}$ & $\begin{array}{c}\text { Frecuencias } \\
\text { absolutas }\end{array}$ & $\begin{array}{c}\text { Frecuencias } \\
\text { Relativas }\end{array}$ \\
\hline $0-\leq 1$ & 1 & 0,003 & 1 & 0,003 \\
$>1-\leq 2$ & 6 & 0,016 & 0 & 0,000 \\
$>2-\leq 3$ & 51 & 0,133 & 2 & 0,005 \\
$>3-\leq 4$ & 106 & 0,276 & 85 & 0,221 \\
$>4-\leq 5$ & 210 & 0,547 & 264 & 0,688 \\
$>5-\leq 6$ & 10 & 0,025 & 32 & 0,083 \\
\hline$\Sigma$ & 384 & 1,0 & 384 & 1,0 \\
\hline
\end{tabular}




\begin{tabular}{|c|c|c|c|c|}
\hline \multirow{2}{*}{$\begin{array}{l}\text { B. Ríos } \\
\qquad \begin{array}{l}\text { Clases } \\
\text { (Log10) }\end{array}\end{array}$} & \multicolumn{2}{|c|}{ Puntos localidades verdaderas } & \multicolumn{2}{|c|}{ Puntos aleatorios } \\
\hline & $\begin{array}{c}\text { Frecuencias } \\
\text { absolutas }\end{array}$ & $\begin{array}{c}\text { Frecuencias } \\
\text { relativas }\end{array}$ & $\begin{array}{c}\text { Frecuencias } \\
\text { absolutas }\end{array}$ & $\begin{array}{c}\text { Frecuencias } \\
\text { Relativas }\end{array}$ \\
\hline $0-\leq 1$ & 2 & 0,005 & 2 & 0,005 \\
\hline$>1-\leq 2$ & 9 & 0,023 & 3 & 0,008 \\
\hline$>2-\leq 3$ & 72 & 0,188 & 24 & 0,063 \\
\hline$>3-\leq 4$ & 187 & 0,487 & 197 & 0,513 \\
\hline$>4-\leq 5$ & 114 & 0,297 & 158 & 0,411 \\
\hline$\Sigma$ & 384 & 1,0 & 384 & 1,0 \\
\hline C. Vías & \multicolumn{2}{|c|}{ Puntos localidades verdaderas } & \multicolumn{2}{|c|}{ Puntos aleatorios } \\
\hline Clases & Frecuencias & Frecuencias & Frecuencias & Frecuencias \\
\hline$(\log 10)$ & absolutas & relativas & absolutas & Relativas \\
\hline $0-\leq 1$ & 3 & 0,008 & 0 & 0,000 \\
\hline$>1-\leq 2$ & 32 & 0,083 & 3 & 0,008 \\
\hline$>2-\leq 3$ & 133 & 0,346 & 37 & 0,096 \\
\hline$>3-\leq 4$ & 129 & 0,336 & 163 & 0,424 \\
\hline$>4-\leq 5$ & 84 & 0,219 & 172 & 0,448 \\
\hline$>5-\leq 6$ & 3 & 0,008 & 9 & 0,024 \\
\hline$\Sigma$ & 384 & 1,0 & 384 & 1,0 \\
\hline
\end{tabular}

en la que a cada celda se le asigna un peso al promediar los valores de puntos cercanos de la muestra que está siendo analizada (registros). Mientras más cercano es un punto a la celda que esta siendo procesada, mayor influencia o peso le es asignada. Para este proceso se fijó la búsqueda de 12 puntos o registros de colección más cercanos en celdas de 4,6 km por lado.

Las áreas del mapa donde existen celdas a las que se les ha asignado un valor alto serán aquellas en donde hay un esfuerzo de muestreo mayor mientras que aquellas zonas donde las celdas han sido asignadas con valores bajos porque en ese lugar, o su vecindad, el esfuerzo de muestreo ha sido menor, por lo tanto, estas zonas serán propuestas como zonas prioritarias de

\section{colección.}

\section{RESULTADOS Y DISCUSIÓN}

Los ejemplares que componen la colección de mamíferos del QCAZ han sido colectados preferentemente cerca de centros poblados, ríos o vías. En todos los casos, las distancias de las localidades a los poblados, ríos y vías son significativamente diferentes, y más cercanas, que las distancias de los puntos aleatorios que se utilizaron como artefacto de comparación con los resultados observados (Tabla 1).

La prueba estadística de KolmogorovSmirnov para el análisis de la cercanía a centros poblados demostró que la distribución de las distancias de registros de localidades de colección es significativamente 
diferente a la distribución de distancias de puntos aleatorios $(\mathrm{z}=3,067 ; \mathrm{p}<0,001)$. En este caso se puede observar que el $15,2 \%$ de especímenes han sido colectados entre $0-1 \mathrm{~km}$, frente a $0,78 \%$ de puntos aleatorios ubicados en el mismo rango de distancia. Como compensación, se puede observar que una proporción del 54,7 \% de los registros de localidades caen entre 10 $100 \mathrm{~km}$ de distancia al poblado más cercano, cuando la proporción que se espera si las colecciones fueran regulares es de $68,8 \%$ (Tabla 1A). Un resultado similar fue encontrado para el análisis de cercanía a ríos, donde la proporción de localidades entre $0-1 \mathrm{~km}$ asciende a $21,6 \%$ frente a $7,6 \%$ de puntos aleatorios. A pesar de no existir una amplia diferencia entre los registros de colección ubicados entre 10 $100 \mathrm{~km}$ de distancia del río más cercano $(48,7 \%)$ y los registros esperados si las colecciones fueran planificadas regularmente $(51,3 \%)$, la proporción de ejemplares encontrados a menos de $1 \mathrm{~km}(21,6 \%)$ hace que la diferencia de la distribución de registros de localidades observados frente a los esperados sea altamente significativa $(\mathrm{z}=2,815 ; \mathrm{p}<0,001)$, observándose que una proporción importante de localidades están claramente más cercanos a sistemas fluviales (Tabla 1B).

Por último, resultados similares se obtuvieron con el análisis de cercanía a vías. Se encontró que el 43,7\% de las localidades registradas se ubican a distancias menores de un kilómetro frente a una proporción del 10,4\% de resultados esperados, para el mismo rango de distancia. Estos resultados reflejan, al igual que en los otros casos, que la diferencia en la distribución de los registros de localidades es altamente significativa $(\mathrm{z}=4,763 ; \mathrm{p}<0,001)$ respecto a la distribución de distancias de los puntos aleatorios y que las distancias reales son claramente menores que aquellas de puntos aleatorios (Tabla 1C).

Ya que sin duda los centros poblados, ríos y vías son medios de accesibilidad hacia lugares de interés para realizar colecciones científicas, el muestreo de mamíferos está ampliamente sesgado a dichos medios, habiendo una mayor inclinación a realizar colecciones muy cerca de vías. Esta es una característica particular de colecciones biológicas, encontrada en estudios previos $(4,5)$.

Sin embargo, realizar los análisis de proximidad tomando la cercanía a ríos, vías y poblados por separado, podría subestimar los sesgos reales que podrían existir en una colección científica, ya que el rasgo geográfico cercano al registro podría estar asociado solamente a una de las tres capas mencionadas. El resultado del análisis de cada punto de colección al rasgo geográfico más cercano, independientemente de que sea río, vía o poblado, confirma que existe preferencia hacia colectar ejemplares en las cercanías de lugares fácilmente accesibles para los colectores (Tabla 2). La prueba estadística de Kolmogorov-Smirnov determinó que la distribución de las distancias de registros de localidades de colección es significativamente diferente a la distribución de distancias de puntos aleatorios al poblado, río $\mathrm{o}$ vía más cercanos $(\mathrm{z}=5,846 ; \mathrm{p}<0,001)$.

Se puede notar que el $61,45 \%$ de los registros de la colección de mamíferos del QCAZ han sido colectados a distancias de entre $0-1 \mathrm{~km}$ de algún centro poblado, río o carretera; triplicando el porcentaje de 
Tabla 2. Cantidad y proporción de localidades de colección y puntos aleatorios en los diferentes rangos de distancias al rasgo geográfico más cercano. Las clases representan rangos de distancias transformadas a su logaritmo natural $(>0-\leq 1=>0 \mathrm{~m}-\leq 10 \mathrm{~m} ;>1-\leq 2=>10 \mathrm{~mm}-\leq 100 \mathrm{~m} ;>2-\leq 3=>100 \mathrm{~m}-\leq 1 \mathrm{~km}$; $>3-\leq 4=>1 \mathrm{~km}-\leq 10 \mathrm{~km} ;>4-\leq 5=>10 \mathrm{~km}-\leq 100 \mathrm{~km} ;>5-\leq 6=>100 \mathrm{~km})$.

\begin{tabular}{|ccccc|}
\hline & \multicolumn{2}{c}{ Puntos localidades verdaderas } & \multicolumn{2}{c|}{ Puntos aleatorios } \\
\hline Clases & $\begin{array}{c}\text { Frecuencias } \\
\text { absolutas }\end{array}$ & $\begin{array}{c}\text { Frecuencias } \\
\text { relativas }\end{array}$ & $\begin{array}{c}\text { Frecuencias } \\
\text { absolutas }\end{array}$ & $\begin{array}{c}\text { Frecuencias } \\
\text { Relativas }\end{array}$ \\
\hline $0-\leq 1$ & 4 & 0,010 & 0 & 0,000 \\
$>1-\leq 2$ & 74 & 0,193 & 13 & 0,034 \\
$>2-\leq 3$ & 158 & 0,411 & 68 & 0,177 \\
$>3-\leq 4$ & 130 & 0,339 & 231 & 0,602 \\
$>4-\leq 5$ & 18 & 0,047 & 72 & 0,188 \\
\hline$\Sigma$ & 384 & 1,0 & 384 & 1,0 \\
\hline
\end{tabular}

registros que se esperaría si la colección fuese regular en el territorio nacional. Esto se apoya en lo pobremente representadas que se encuentran las colecciones entre 10 - $100 \mathrm{~km}$ de distancia a un poblado, río o vía $(4,68 \%$ frente al $18,75 \%$ esperado (Tabla 2).

Con el fin de fortalecer el análisis de los resultados obtenidos, se proyectaron las localidades de colección en el mapa de Accesibilidad del Ecuador del Proyecto BINU (13), lo que permitió observar que gran parte de los ejemplares han sido colectados en zonas que quedan a pocas horas de viaje desde un lugar de fácil acceso como centros poblados, vías, ríos, aeropuertos, etc. (Figura 3). Una comparación numérica de las localidades de colección y los puntos aleatorios generados con SIG, demuestra que aproximadamente el 64,8\% de los ejemplares han sido colectados en lugares a los que toma llegar entre 0 y 1 hora de viaje desde cualquier lugar accesible, mientras que la proporción esperada, de acuerdo a los puntos aleatorios es de únicamente la tercera parte del total de registros.

Estos resultados revelan la importancia de diseñar muestreos sistemáticos que encuentren la forma de acceder a zonas no muestreadas por ser de difícil acceso y que han sido previamente propuestos como

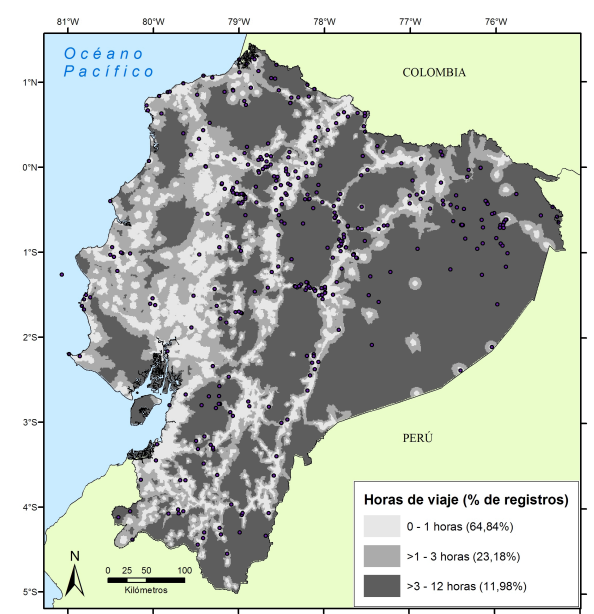

Figura 3. Localidades de colección proyectadas en el mapa de accesibilidad del Ecuador. Se puede observar que la mayoría de localidades se encuentran en zonas de fácil acceso. 
hábitats apropiados para la localización de especies de interés (12).

Según un estudio de Balmford et al. (20), la riqueza de aves, mamíferos, serpientes y anfibios en África está positivamente correlacionada con una alta densidad de población humana, y mencionan que este patrón puede estar generalizado en toda la región tropical. Dicho estudio analiza grupos ampliamente muestreados y su relación con áreas humanas densamente pobladas; sin embargo, no analiza sesgos geográficos de colección. Independientemente, Reddy y Dávalos (6) atribuyen los resultados de Balmford y colaboradores a la preferencia de los investigadores por hacer colecciones en zonas más accesibles y a cortas distancias de zonas habitadas y no necesariamente porque la diversidad de vertebrados está relacionada directamente con lugares más densamente poblados. Los resultados encontrados en el presente trabajo coinciden con los de Reddy y Dávalos, demostrando las preferencias de los colectores a realizar capturas en lugares de fácil acceso.

A diferencia de un estudio realizado en África, donde se evidencian preferencias al realizar colecciones biológicas dentro de áreas protegidas o de prioridad para la conservación (6), el presente trabajo arrojó

Tabla 3. Análisis estadístico para sesgo en la colección del QCAZ frente a los registros esperados si éstos fueran colectados según la proporción de territorio nacional ocupado por el SNAP y áreas no protegidas.

\begin{tabular}{|cccccc|}
\hline $\begin{array}{c}\text { Probabilidad } \\
\text { Resultados } \\
\text { Observados }\end{array}$ & $\begin{array}{c}\text { Resultados } \\
\text { de ocurrencia } \\
\mathrm{P}\end{array}$ & $\begin{array}{c}\text { Esperados } \\
\mathrm{np}\end{array}$ & $\begin{array}{c}\text { Ji cuadrado } \\
\left({ }^{2}\right)\end{array}$ & Significación & \\
\hline $\begin{array}{c}\text { Ejemplares } \\
\text { dentro SNAP } \\
\text { Ejemplares } \\
\text { fuera SNAP }\end{array}$ & $696(13,05 \%)$ & 0,162 & 1236,55 & 55,83 & $* *$ \\
\hline $\mathrm{n}$ & $7637(86,95 \%)$ & 0,838 & 6396,45 & & $\mathrm{p}<0,01$ \\
\hline
\end{tabular}

Tabla 4. Análisis estadístico para sesgo en los registros del QCAZ frente a la cantidad de registros esperados arrojados por los puntos aleatorios generados por herramientas de SIG.

\begin{tabular}{|c|c|c|c|c|}
\hline & $\begin{array}{l}\text { Resultado } \\
\text { Observados }\end{array}$ & $\begin{array}{l}\text { Esperados } \\
\text { (aleatorios) }\end{array}$ & $\begin{array}{c}\text { Resultados } \\
\text { Ji cuadrado } \\
\quad\left({ }^{2}\right)\end{array}$ & Significación \\
\hline $\begin{array}{l}\text { Ejemplares } \\
\text { dentro SNAP }\end{array}$ & $996(13,05 \%)$ & 1233 & \multirow{2}{*}{54,32} & 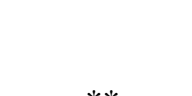 \\
\hline $\begin{array}{l}\text { Ejemplares } \\
\text { fuera SNAP }\end{array}$ & $6637(86,95 \%)$ & 6400 & & $\mathrm{p}<0,01$ \\
\hline $\mathrm{n}$ & 7633 & 7633 & & \\
\hline
\end{tabular}


resultados contrarios. Un primer análisis de Ji cuadrado determinó que los esfuerzos de muestreo en el territorio nacional continental no están regularmente distribuidos según la proporción de territorio ocupado por el SNAP y áreas no protegidas. Se encontró que existe una diferencia altamente significativa $\left({ }^{2}=55,83 ; \mathrm{p}<0,01\right)$ en la proporción de especímenes colectados dentro $(13,05 \%)$ y fuera del SNAP $(86,95 \%$; Tabla 3).

Una segunda prueba estadística para determinar si existe un sesgo al realizar colecciones dentro del SNAP utilizando puntos aleatorios, como artefacto de comparación, mostró que existen, igualmente, diferencias altamente significativas $\left({ }^{2}=54,32\right.$; $\mathrm{p}<0,01)$ entre la cantidad de registros colectados y registrados en la colección de mamíferos del QCAZ, frente a la cantidad de registros esperados arrojados por los pun-

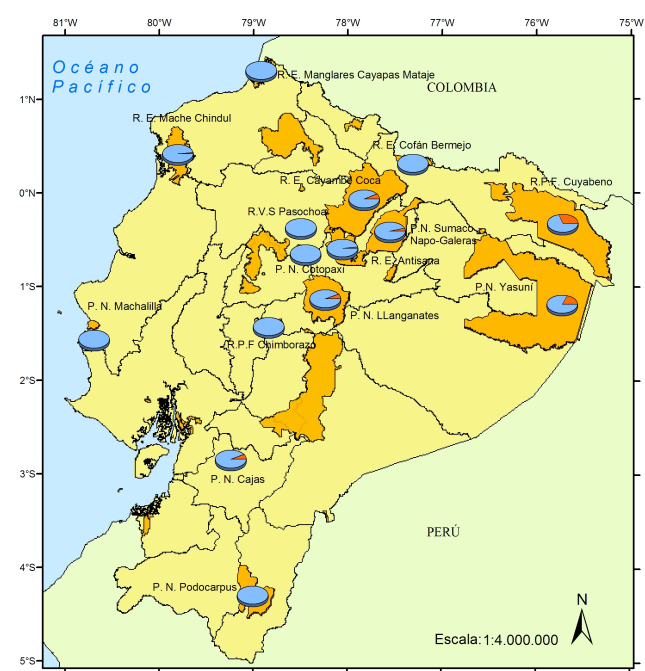

tos aleatorios generados (Tabla 4).

Una de las características de las zonas que conforman el SNAP es que albergan gran riqueza y diversidad (11), sin embargo presentan, según el presente análisis, un esfuerzo de muestreo desproporcionadamente menor, lo que no es una característica de las colecciones biológicas. (6) Las zonas que muestran poco o ningún esfuerzo de muestreo coinciden con áreas protegidas, al igual que lo recalcara Apezteguia (12). En el QCAZ, 20 de las 35 áreas protegidas no tienen ningún registro de colección constituyendo un vacío importante en el conocimiento de la mastofauna ecuatoriana.

La Figura 4 representa las áreas del SNAP en las que se han concentrado los esfuerzos de muestreo del QCAZ, aquellas que presentan menos registros son: Parque Nacional Cotopaxi, Parque Nacional
1. Parque Nacional Cajas
2. Parque Nacional Cotopaxi
3. Parque Nacional Llanganates
4. Parque Nacional Machalilla
5. Parque Nacional Podocarpus
6. Parque Nacional Sumaco-Napo Galeras
7. Parque Nacional Yasuní
8. Reserva Ecológica Antisana
9. Reserva Ecológica Cayambe-Coca
10. Reserva Ecológica Cofán-Bermejo
11. Reserva Ecológica Mache Chindul
12. Reserva Ecológica Manglares Cayapas Mataje
13. Reserva de Producción Faunística Chimborazo
14. Reserva de Producción Faunística Cuyabeno
15. Reserva de Vida Silvestre Pasochoa

Figura 4. Mapa del Ecuador indicando el esfuerzo de muestreo diferencial en las zonas del SNAP del territorio continental. Se indican las áreas protegidas que tienen más de un registro de colección y se indica, con gráficos de pie, la proporción de ejemplares colectados del total de registros del QCAZ en las zonas del SNAP 
Machalilla, Parque Nacional Podocarpus, Reserva Ecológica Cofán-Bermejo, Reserva Ecológica Manglares Capayas Mataje, Reserva de Producción Faunística Chimborazo, Reserva de Vida Silvestre Pasochoa, Reserva Ecológica Mache Chindul y la zona sur del Parque Nacional Yasuní.

Actualmente, y en vista de todos los problemas de conservación, la principal razón para la designación de áreas protegidas es la representación de todos los niveles de biodiversidad. $(11,12)$ Sin embargo, en el Ecuador, la falta de colecciones en estas áreas hace que su designación sea, en la mayoría de ellas, incoherente tomando en cuenta que el conocimiento de la biodiversidad debió basarse en análisis de ejemplares en museos y herbarios. Cabe recalcar que es necesario incorporar el análisis de otras colecciones de mamíferos en Ecuador y el exterior para verificar esta tendencia y planificar futuras colecciones en el SNAP.

El sesgo encontrado hacia la limitada colección de ejemplares dentro de áreas protegidas se debe, en parte, a obstáculos operativos y burocráticos en los trámites para la obtención de permisos de investigación que otorga el Ministerio del Ambiente. A este hecho se suman la falta de preparación de algunos funcionarios de este organismo del Estado, el desconocimiento e incorrecta interpretación de las leyes ambientales y la poca concientización sobre la importancia de la investigación en la formulación de estrategias de conservación.

Como resultado del análisis de interpolación, se advierten cuatro zonas donde no existen registros de colección y que se pro-

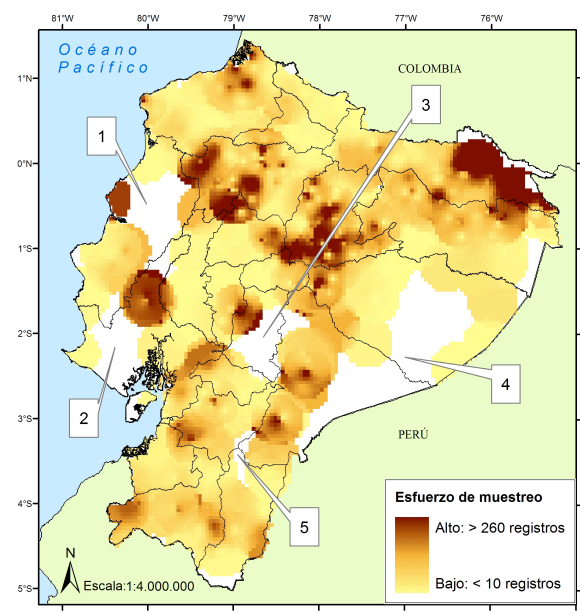

Figura 5. Interpolación de los registros de colección para determinar áreas prioritarias de muestreo

ponen en el presente trabajo como áreas prioritarias de investigación. Estas zonas corresponden a (1) la zona central de la provincia de Manabí; (2) los alrededores de la península de Santa Elena; (3) el Parque Nacional Sangay y sus alrededores; (4) las zonas de la Amazonia comprendidas entre el sur del Parque Nacional Yasuní, las estribaciones orientales de los Andes y la frontera con Perú; y, (5) las zonas cercanas a la cordillera del Cóndor (Figura 5).

Cabe mencionar, además, que es importante realizar muestreos en toda la zona sur del Ecuador. El territorio nacional que comprende las provincias de El Oro, Azuay, Loja y Zamora Chinchipe está pobremente muestreado. Esto se debe, sin duda, a la falta de proyectos de investigación en dicha zona y la distancia de estas provincias a Quito, donde residen y trabajan los investigadores asociados al QCAZ, confirmando la preferencia en hacer colecciones en lugares cercanos y previamente 
visitados y conocidos.

\section{CONCLUSIONES}

En general, los esfuerzos de muestreo de las colecciones biológicas no presentan distribuciones espaciales regulares debido a la preferencia de los investigadores a realizar colecciones cerca de lugares accesibles. La colección de mamíferos del QCAZ está ampliamente sesgada hacia la captura de ejemplares muy cerca de poblados, ríos y vías.

La colección de mamíferos del QCAZ almacena muy pocos ejemplares colectados dentro del SNAP. Si este bajo esfuerzo de muestreo es similar en colecciones biológicas del Ecuador almacenadas en otros museos, el conocimiento de la diversidad de estas zonas sería incompleto resultando poco confiable asegurar que estás áreas han sido designadas para proteger la biodiversidad biológica del país.

Existen zonas en el Ecuador que están pobremente muestreadas y son propuestas en el presente trabajo como zonas prioritarias para realizar colecciones de mamíferos. Estas zonas corresponden a la zona central de la provincia de Manabí, los alrededores de la península en la de Guayas, el Parque Nacional Sangay y sus alrededores y la zona de la Amazonia comprendida entre el sur del Parque Nacional Yasuní y la Cordillera del Cóndor.

\section{LITERATURA CITADA}

1. SOBERÓN, J., DÁVILA, P., GOLUBOV, J. 2003. Targeting Sites for Biological Collections. En: R.D.
Smith, J.B. Dickie, S.H. Linington, H.W. Pritchard y R.J. Probert, Eds. Seed Conservation. Turning Science into Practice. pp. 221-231. Royal Botanic Garden, U.K.

2. O'CONNELL, A.F JR., GILBERT, A.T., HATFIELD, J.S. 2004. Contribution of Natural History Collection Data to Biodiversity Assessment in National Parks. Conservation Biology 18 (5):12541261.

3. GREEN, K. 1999. Development of the Spatial Domain in Resource Management. En: S. Morain, Ed. GIS Solutions in Natural Resource Management: Balancing the TechnicalPolitical Equation. pp. 6-9. On Word Press, Santa Fe, U.S.A.

4. WILLIAMS, P.H., MARGULES, C.R., HILBERT, D.W. 2002. Data requirements and data sources for biodiversity priority area selection. Journal of Bioscience 27(4): 327-338.

5. FREITAG, S., HOBSON, C., BIGGS, H.C., VAN JAARSVELD, A.S. 1998. Testing for potential survey bias: the effect of roads, urban areas and nature reserves on a southern African mammal data set. Animal Conservation 1: 119-127.

6. REDDY, S., DÁVALOS, L.M. 2003. Geographical sampling bias and its implications for conservation priorities in Africa. Journal of Biogeography 30: 1719-1727.

7. ROMO, H., GARCÍA-BARROS, E., LOBO, J.M. 2006. Identifying recorder-induced geografic bias in an Iberian butterfly database. Ecography 29(6): 873-885. 
8. GUISAN, A., ZIMMERMANN, N.E. 2000. Predictive habitat distribution models in ecology. Ecological Modelling 135: 147-186.

9. RAXWORTHY, C. J., MARTÍNEZMEYER, E., HORNING, N., NUSSBAUM, R. A., SCHNEIDER, G. E., ORTEGA-HUERTA, M., PETERSON,

A. T. 2003. Predicting distributions of known and unknown reptile species in Madagascar. Nature 426: 837 - 841.

10. PHILLIPS, S., ANDERSON, R. P., SCHAPIRE, R. E. 2006. Maximum entropy modeling of species geographic distributions. Ecological Modelling 190:231-259.

11. MINISTERIO DEL AMBIENTE. Ecuador Megadiverso. Áreas protegidas, [en línea]. Ministerio del Ambiente de la República del Ecuador. (2006). Dirección URL: <http://www.ambiente.gov.ec>. [Consulta: 17 de enero 2007].

12. APEZTEGUIA, A. 2006. Elaboración de modelos de distribución geográfica de micromamíferos voladores y uso de herramientas de Sistemas de Información Geográfica para priorizar áreas de investigación en la Amazonía del Ecuador. Tesis de Licenciatura, Pontificia Universidad Católica del Ecuador, Quito, Ecuador.

13. UNEP-WORLD CONSERVATION MONITORING CENTER. 2005. En Indicadores de Biodiversidad para Uso Nacional (Proyecto BINU), [CDROM]. Fundación Ecociencia y
Ministerio del Ambiente.

14. ALIANZA JATUN SACHA - CDCECUADOR. 2002. Centros poblados a nivel nacional. En Almanaque Electrónico Ecuatoriano, [CDROM]. Alianza Jatun Sacha - CDCEcuador,

15. ENVIRONMENTAL SYSTEMS RESEARCH INSTITUTE. 2001. ArcGIS $^{\mathrm{TM}}$ Desktop v. 8.1. Environmental Systems Research Institute, Inc., New York, U.S.A.

16. SOKAL, R.R, ROHLF, F.J. 2003. Biometry: the principles and practice of statistics in biological research. Tercera edición. W. H. Freeman and Company, New York, U.S.A.

17. SÁNCHEZ, J. 2005. Introducción a la estadística no paramétrica y al análisis multivariado. Quality Print, Quito, Ecuador.

18. SPIEGEL, M. 1991. Estadística. Segunda edición. Mc Graw-Hill, México D.F., México.

19. SÁNCHEZ, J. 2004. Introducción a la estadística en las ciencias biológicas. Quality Print, Quito, Ecuador.

20. BALMFORD, A., MOORE, J.L., BROOKS, T., BURGESS, N., HANSEN, L.A., WILLIAMS, P., RAHBEK, C. 2001. Conservation Conflicts Across Africa. Science 291: 2616-2619.

21. PRESSEY, R.L. 1994. Ad Hoc Reservations: Forward or Backward Steps in Developing Representative 